\title{
High-Resolution Laser Doppler Measurements of Microcirculation in the Deep Brain Structures: A Method for Potential Vessel Tracking.
}

Karin Wårdell, Simone Hemm-Ode, Peter Rejmstad and Peter Zsigmond

Linköping University Post Print

\section{Tweet}

N.B.: When citing this work, cite the original article.

Original Publication:

Karin Wårdell, Simone Hemm-Ode, Peter Rejmstad and Peter Zsigmond, High-Resolution Laser Doppler Measurements of Microcirculation in the Deep Brain Structures: A Method for Potential Vessel Tracking., 2016, Stereotactic and Functional Neurosurgery, (94), 1, 1-9. http://dx.doi.org/10.1159/000442894

Copyright: Karger

http://www.karger.com/

Postprint available at: Linköping University Electronic Press

http://urn.kb.se/resolve?urn=urn:nbn:se:liu:diva-124691 


\title{
High Resolution Laser Doppler Measurements of Microcirculation in the Deep Brain Structures - a Method for Potential Vessel Tracking
}

\author{
Karin Wårdell ${ }^{1}$, MSc, PhD, Simone Hemm-Ode ${ }^{1,2}$, MSc, PhD, Peter Rejmstad ${ }^{1}$, MSc and Peter \\ Zsigmond3, MD, PhD \\ ${ }^{1}$ Department of Biomedical Engineering, Linköping University, Sweden \\ ${ }^{2}$ University of Applied Sciences and Arts Northwestern Switzerland \\ School of Life Sciences, Institute for Medical and Analytical Technologies, Switzerland \\ ${ }^{3}$ Department of Neurosurgery and Department of Clinical and Experimental Medicine, Linköping \\ University, Linköping, Sweden
}

Running title: Microcirculation in deep brain structures

Key words: deep brain stimulation (DBS), laser Doppler flowmetry (LDF), stereotactic neurosurgery, microcirculation, navigation

\section{Corresponding author:}

Karin Wårdell

Dept. of Biomedical Engineering

Linköping University

58185 Linköping

Phone: +46 101030000

Email: karin.wardell@liu.se 


\section{Abstract}

BACKGROUND: Laser Doppler flowmetry (LDF) can be used to measure cerebral microcirculation in relation to stereotactic deep brain stimulation (DBS) implantations.

OBJECTIVE: To investigate the microcirculation and total light intensity (TLI) corresponding to tissue greyness in DBS-target regions with high resolution LDF-recordings, and to define a resolution which enables detection of small vessels.

METHODS: Stereotactic LDF-measurements were done prior to DBS-implantation with $0.5 \mathrm{~mm}$ steps in the vicinity to four deep brain targets (STN, GPi, Vim, Zi) along 20 trajectories. MannWhitney-U-test was used to compare the microcirculation and TLI between targets, and the measurement resolution ( 0.5 vs. $1 \mathrm{~mm})$. The number of high blood flow spots along the trajectories were calculated.

RESULTS: There was a significant difference $(\mathrm{p}<0.05)$ in microcirculation between the targets. High blood flow spots were present at 15 out of 510 positions, seven along Vim and GPi trajectories respectively. There was no statistical difference between resolutions even though both local blood flow and TLI-peaks could appear at $0.5 \mathrm{~mm}$ steps.

CONCLUSIONS: LDF can be used for on-line tracking of critical regions presenting blood flow and TLI-peaks, possibly relating to vessel structures and thin laminas along stereotactic trajectories. 


\section{Introduction}

The clinical indications and use of deep brain stimulation (DBS) are constantly increasing, and thus there is a need for safe and efficient surgical implantation procedures and further understanding of the mechanism of action [1-3]. Improved magnetic resonance imaging (MRI), often tailored to specific DBS-targets, has increased the possibilities to do direct brain targeting in the surgical planning phase [4]. However, still many centers perform different types of intraoperative recordings in order to make the final decision on trajectory or target position.

Microelectrode recording (MER) is one of the most common intraoperative registration methods and allows for measurements in the vicinity to the preplanned target region. As such, neuronal measurements can be done in up to five tracks with an unfortunate increased risk of bleedings [5, 6]. This is dependent on several factors among these the choice of trajectory path, number of trajectories and target region. A possible additional risk factor could be the very thin MERelectrode tip ( $\varnothing \sim 0.1 \mathrm{~mm}$ ) which may prick small vessels during the insertion. Impedance recording [7] is another established intraoperative technique which can support the surgeon to define the final target e.g. through a sound signal in the operating theater. Impedance probes are often combined with radio frequency (RF) lesioning features, and as so they have a relatively broad probe tip ( $\varnothing \sim 1-2 \mathrm{~mm}$ ) compared to the MER-electrodes. Such probe designs may have the advantage of reducing the risks of bleedings, but on the other hand the larger probe surface also results in a lower measurement resolution due to the spread into a larger tissue volume. Optical techniques are possible alternatives for intraoperative recordings during stereotactic neurosurgery. Optical probes with similar outer dimensions as used for impedance measurements have previously been designed and evaluated during DBS-surgery [8, 9]. By integrating forward looking optical fibers along the probe shaft the sample volume $[10,11]$ can be kept more precise than for impedance. In a study where a probe was designed for combined stereotactic impedance and optical measurements it was shown that the optical technique was superior to the impedance method regarding tissue type estimation [12].

Previous studies using diffuse reflectance spectroscopy [13-16] and laser Doppler flowmetry (LDF) $[9,17]$ have shown that the total light intensity (TLI) backscattered from brain tissue can be used to distinguish between different shades of grey and white matter, and thus different 
structures passed during stereotactic probe insertion. The original application of LDF, however, is to measure the tissue's microcirculation $[18,19]$. Over the years many preclinical and clinical studies of microcirculation in the skin, kidney, muscle, bone, heart etc. have been performed, but only a few studies have been accomplished in the human brain. The latter is most probably due to the complex clinical measurement situation and possible the techniques inherent sensitivity for movement artifacts. However, when LDF is used together with a stereotactic frame this obstacle is overcome, and LDF has shown a great potential as intraoperative monitoring device in relation to stereotactic DBS-implantations as both the microcirculation and TLI can be recorded simultaneously. Recently, the relation between TLI and microcirculation, and anatomy was explored in detail for two common DBS-targets; the subthalamic nucleus (STN) and the ventral intermediate nucleus (Vim) [17]. In that study TLI and microcirculatory blood flow were recorded by means of a mechanical device along the entire trajectory starting from the cortex. From these measurements, which were done at $1 \mathrm{~mm}$ steps, optical data i.e. the shades of grey and microcirculation could be directly related to structures passed by means of the respective patients own MRI, planned trajectory and a surgical planning system.

The present study aims at investigating the cerebral microcirculation and shades of gray matter in the same region as MER is usually performed i.e. the last $10 \mathrm{~mm}$ from the preplanned stereotactic targets. The resolution was increased from $1 \mathrm{~mm}$ to $0.5 \mathrm{~mm}$ and recordings done in the vicinity of twenty trajectories towards four different targets, in order to find a resolution that can detect small vessel structures.

\section{Material and Methods}

\section{Patients}

Eleven patients (four female and seven male) ages 43 to 77 (mean (m) \pm standard deviation (s.d.) $66 \pm 9$ years) referred for unilateral or bilateral DBS-implantation at the Department of Neurosurgery, Linköping University Hospital were included in the study. In total twenty leads were implanted; eight in STN, six in Vim, four in the globus pallidum internus (GPi) and two in the zona incerta (Zi). The study was approved by the local ethics committee at the University Hospital in Linköping (D. no. M182-04, T54-09) and informed written consent was received from the patients. 


\section{Imaging and surgical procedure}

The surgical procedures followed the protocol for routine DBS-implantation at the clinic and were done under general anaesthesia for the STN, Zi, GPi-targets and as awake surgery for the Vim targets. A stereotactic CT (slice thickness $1 \mathrm{~mm}$, GE Lightspeed Ultra, GE Healthcare, UK) was performed the day of surgery with the Leksell ${ }^{\circledR}$ Stereotactic System (LSS, Model G, Elekta Instrument AB, Sweden). Direct anatomical targeting of the STN, Zi and GPi were done on stereotactic $1.5 \mathrm{~T}$ or 3T MRI (T1 and T2, slice thickness $2 \mathrm{~mm}$, Philips Intera, The Netherlands) or after fusion with the stereotactic CT in Leksell Surgiplan ${ }^{\circledR}$ (Elekta Instrument AB, Sweden). The Schaltenbrandt atlas coordinates for the Vim nucleus were used; 5-7 mm anterior to the posterior commissure, at the level of the intercommissural line, and 13-15 mm lateral to the midline of the $3^{\text {rd }}$ ventricle.

In order to create the trajectories for the respective DBS-electrodes, the probe for intraoperative optical measurements which also acted as guide for the DBS electrodes, was inserted step-vise with the help of a LSS-compatible mechanical device [17]. Recorded optical signals were presented online in the operating theatre, and stored for statistical analysis. When reaching the target area, the probe's position was verified intra-operatively with fluoroscopy (Philips BV Pulsera, Philips Medical Systems, The Netherlands) and then replaced by the DBS electrode (Lead 3389, Medtronic Corporation, Minneapolis, MN, USA). The pulse generator and extension cable were implanted under general anesthesia at the same surgical session. The entire procedure took 4-5 hours of which the optical measurements took 10-15 minutes per side. A postoperative CT scan was performed in order to confirm the electrode positions.

\section{Intraoperative optical measurement}

A laser Doppler flowmeter (PF 5000, Perimed AB Sweden) equipped with a $780 \mathrm{~nm}$ laser with a power of $1 \mathrm{~mW}$ was used for the study. The software developed in LabVIEW (National Instruments, Inc., TX, USA), presented the recorded signals on-line in the operation theatre, and also made postoperative data analysis possible. The optical probe's outer dimensions (length = $190 \mathrm{~mm}, \varnothing=2.2 \mathrm{~mm}$ except for the last $30 \mathrm{~mm}$ where $\varnothing=1.5 \mathrm{~mm}$ ) were adapted to the LSS. The LDF was used for measurements of the local microcirculation (range 0 - 999 arbitrary units [a.u.], also denoted perfusion and blood flow) and tissue type (white or gray matter and shades of grey matter) by means of the TLI signal (range 0 - 10.0 a.u.). Perfusion and TLI levels were 
checked in Motility Standard (Perimed AB, Sweden) before and after the measurement sessions in order to ascertain comparable levels in-between measurements. Correction was done if the recorded levels deviated from the calibration settings. Before surgery, the probe was sterilized according to the STERRAD ${ }^{\circledR}$ protocol [20].

Recordings, each one lasting for 5-30 s, were performed in the cortex and at 40, 20 and $10 \mathrm{~mm}$ from the target region with LDF (Fig. 1). Thereafter LDF-measurements were done,-in $0.5 \mathrm{~mm}$ steps until the pre-calculated target was reached. Along six STN trajectories, signals were captured 1-3 mm beyond the target. Movements of a hand-controlled positioning screw on the mechanical device fixed on the LSS gave rise to a pronounced artifact in the perfusion signal. This artifact was used as position reference for the data analysis. In total, recordings were done at 510 sites.

\section{Analysis}

\section{Target verification}

The preoperative MRI and postoperative CT images were both co-registered, and fused in Leksell Surgiplan ${ }^{\circledR}$ in order to determine the electrode position as well as the respective trajectory. The Schaltenbrand brain atlas was superimposed when necessary in order to identify anatomical structures. Notes were taken of deviations and anatomical structures in the target region. These were also compared to the fluoroscopy images captured during surgery in order to verify the target point. If necessary, the optical targets were adjusted to the target points according to fluoroscopy.

\section{Microcirculation}

Postoperatively, the microcirculation and TLI were calculated for all measurement sites as an average including a time span of recordings covering 5-10 s. This was done using the LabView software by identifying the artifacts in the perfusion signal arising due to the mechanical device movement. Along one STN trajectory the perfusion signal was close to zero at all sites, and data from this trajectory therefore excluded in the statistical analysis. Plots representing the perfusion and TLI-values against measurement site for the last $10 \mathrm{~mm}$ were created. The curves were sorted into four groups; STN, Vim, Zi and GPi. Mean and s.d. were calculated for the last 10, 5, 2.5 and $1.5 \mathrm{~mm}$ along the respective trajectories as well as median in the respective targets. Targets were 
defined as the average recorded at the positions $+0.5 \mathrm{~mm}, 0 \mathrm{~mm}$ and $-0.5 \mathrm{~mm}$. When applicable the same entities were calculated for values recorded beyond the STN-target.

Mann-Whitney-U-test was used to compare the microcirculation in different regions (10, 5, 2.5 $\mathrm{mm}$ in front of the target and in the target regions themselves), and for different resolutions (0.5 mm vs. 1mm). This was performed in Vim, STN and GPi but not for Zi due to the low number of trajectories. The number of high perfusion spots ( $>100$ a.u.) was identified and at these recordings the peak-to-peak amplitude (P-P) and heart rate (HR) were calculated as well. HR was estimated from the captured LDF-signals using spectral analysis and compared with the one monitored in the operating theatre. Sites with pulsatile blood flow pattern such as vasomotion were notified. MATLAB ${ }^{\circledR}$ (The MathWorks, Inc., Natick, MA, USA) was used for the statistical tests.

\section{Results}

The implanted DBS leads reached the preplanned target in all cases except for two leads. In the patient operated on in the $\mathrm{Zi}$, the left lead was $6 \mathrm{~mm}$ medial and thus touched the lateral thalamic border and also went through the red nucleus (Fig. 2a). This is seen as $\underline{\underline{a}}$ two dips in the TLI signal displaying the considerably darker grey matter compared to the surrounding tissue, compared to the trajectory following the planned path on the right side (Fig. 2c). The corresponding microvascular blood flow values as recorded with $0.5 \mathrm{~mm}$ resolution the last 10 $\mathrm{mm}$ from the target are presented in Fig. 2b. In another patient, a deviation of about $1.5 \mathrm{~mm}$ medial of the preplanned target was found on the second implantation side.

\section{Microcirculation}

High microvascular blood flow spots (> 100 a.u.) were present at 15 sites, seven along Vim and GPi trajectories respectively, and one beyond STN. Of these spots two showed perfusion higher than 300 a.u. along one GPi trajectory (Fig. 3). An example of a very local high blood flow spot with high P-P compared to the surrounding sites was seen $2 \mathrm{~mm}$ beyond an STN target (Fig. 4). This became visible due to the $0.5 \mathrm{~mm}$ measurement resolution. Calculated average perfusion, PP and HR from each measurement spot around the STN target are presented in Fig. 4. It is clearly 
seen how the microcirculation changes step by step, but the calculated HR remains around 54 beats per minute for all sites.

There was a significant difference $(\mathrm{p}<0.05$, Table 1$)$ in microvascular blood flow but not in TLI between the different targets (Fig. 5). Lowest blood flow was found in GPi $(12.8 \pm 4.8, n=9)$ and highest in Vim (30.2 $\pm 19.8, \mathrm{n}=12)$. The blood flow in STN was $19.5 \pm 7.3(\mathrm{n}=23)$ with an increase to $29.9 \pm 22.9(n=45)$ the $3 \mathrm{~mm}$ recordings proximal of the target, i.e. when the probe was reaching the substantia nigra. Significant levels for all analyzed regions are presented in Table 1.

\section{Resolution}

Statistically there was no difference neither for microvascular blood flow or TLI when the measurement resolution was set to $0.5 \mathrm{~mm}$ or $1 \mathrm{~mm}$. However, visually, there was a difference, and both, perfusion and TLI peaks could appear even at $0.5 \mathrm{~mm}$ distance change. Examples are shown in Fig. 4 for the microvascular recording in the vicinity to an STN target, and in Fig. 6 for TLI. In Fig. 6 both the TLI and blood flow are presented. At one spot $50 \mathrm{~mm}$ from the target the TLI was very low at the same time as there was high blood flow. The blood flow increased during the approximately $30 \mathrm{~s}$ long recording and presented a possible vasomotion pattern. Inspection of the MRI showed that the probed passed a sulci. In the target region the TLI showed a peak $0.5 \mathrm{~mm}$ beyond the pre-calculated target (Fig. 6), possibly visualizing the reflectance from a very thin lamina in the thalamus.

\section{Discussion}

In this study the microcirculation was investigated in the deeper part of the brain in eleven patients undergoing DBS-surgery. This was accomplished by a laser Doppler system adapted for use with the Leksell ${ }^{\circledR}$ Stereotactic System. It was found that a resolution of at least $0.5 \mathrm{~mm}$ is necessary to detect local microvascular changes i.e. high perfusion spots, and possibly also thin laminas in the individual patient's brain.

Monitoring of the brain microcirculation with LDF prior to DBS-implantations may help prevent hemorrhage [17]. This is especially important during MER as the number of penetrations together 
with sulcal or ventricular involvements are considered as major surgical risk factors during DBSimplantations [6]. Therefore the present focus was set on the target regions, i.e. the last $10 \mathrm{~mm}$ along the pre-calculated trajectory, corresponding to the distance commonly approached during microelectrode recordings. Also the choice of target itself is considered a risk factor. Higher incidents of hemorrhage compared to STN interventions have been reported in relation to thalamotomy and pallidotomy [21], and in relation to DBS implantations in Vim [22]. In the present LDF-measurements more local blood flow peaks were found both in GPi and Vim regions. The latter agrees with our previous study [17]. The only local blood flow peak in the vicinity of the STN was found exactly $2 \mathrm{~mm}$ beyond the target (Fig. 4). In a similar manner a small TLI peak was seen $0.5 \mathrm{~mm}$ beyond the target in Vim (Fig. 6). As very thin laminas are present in thalamus [23], this local TLI-increase is most probably due to a thin whiter matter stripe. Several of the local peaks presented in this study were only detected due to the high resolution setting i.e. $0.5 \mathrm{~mm}$ measurement distances.

Along one trajectory towards the $\mathrm{Zi}$ the lead was misplaced several millimeters. This is clearly indicated in the fused preoperative MRI and postoperative CT, and the postoperatively generated TLI-curve (Fig. 2). Despite the lead displacement on the left side the patient suffering from severe tremor presented very good clinical results following the DBS-surgery and no reoperation was required. So far the TLI-curves, as presented in this paper, are generated after surgery by manual identification of the movement peaks seen in the perfusion signal. Future software aims at giving a direct feedback of the TLI-curves in relation to the actual measurement position and anatomy. The perfusion data can, however, already today be directly used during the surgical session as an indicator for high perfusion spots, and thus for possible vessel tracking.

Along another trajectory the perfusion values were close to zero and therefore not included in the statistical analysis. This could possibly be caused by dried blood on the probe tip which covers the light and then do not allow for correct processing of the perfusion signals. Alternatively a local vasoconstriction reduced the perfusion signal to even lower values than normal. This is more likely as the TLI signal presented similar values on both sides. In general non-provoked tissue presents perfusion values below 50 a.u. nevertheless there was a significant difference in perfusion between the targets, a finding that also agrees with our previous study were the perfusion was higher in Vim compared to STN [17]. As the intention was to keep surgery as short 
as possible, recordings were excluded along the internal capsula (IC) i.e. the white matter with the strongest TLI. Therefore it was not possible to normalize the TLI signal and extract optical trajectories comparable between individuals. This could also be the reason for the vaguer statistical significance for TLI data compared to our previous study where an average of the maximum intensity values captured in IC were used for normalization [17]. Still, on an individual basis, each TLI-trajectory represents the relative changes of the shades of grey matter.

The interest for photonic brain monitoring and imaging is increasing. Still a majority of the scientific investigations are experimental and originating from animal studies [24, 25]. Few optical techniques have so far reached the clinical setting. One exception is the generic LDF-systems i.e. laser Doppler perfusion imaging [26] and monitoring [18] which have been adapted and also used in neurosurgical theatres during both stereotactic and open surgery [9, 17, 27-29]. Such translations to clinical settings also open up new possibilities for in-depth investigations of cerebral microcirculation especially as some can be performed under awake surgeries when the anesthetic drugs do not influence the tissue bed. Other future possibilities are stimuli-response investigations for instance by combining MER with LDF during DBS-implantations. Such combination techniques may help reduce the risk of hemorrhage in relation to MER as LDF can detect local microvascular perfusion variations intra-operatively.

\section{Conclusion}

Laser Doppler flowmetry is useful for on-line tracking of local blood flow peaks and changes in tissue greyness possible relating to small vessel structures and thin laminas along stereotactic trajectories. A resolution of $0.5 \mathrm{~mm}$ is necessary for discrimination of very locally presented peaks. As the optical is probe is forward looking, this may help reduce bleedings in relation to stereotactic surgery, especially in high risk regions and possible together with microelectrode recordings. 


\section{Acknowledgements}

Johan Richter, MD and the Staff the Department of Neurosurgery, Linköping University Hospital are acknowledged for skillful assistance during the measurements. The study was supported by the Swedish Research Council (621-2013-6078), the Parkinson Foundation at Linköping University and the Swiss National Science Foundation (205321-135285). The authors do not have any conflict of interest to report.

\section{Legends to Figures}

Figure 1. Overview of measurement set up. a) Mechanical insertion device and optical probe positioned in Leksell ${ }^{\circledR}$ Stereotactic System. b) Axial MRI with region of interest marked. c) Sketch of typical path and measurement sites marked. The last $10 \mathrm{~mm}$ i.e. in the vicinity to the target laser Doppler flowmetry measurements were done at $0.5 \mathrm{~mm}$ steps.

Figure 2. a) Fused MR/CT images. Green rings with cross marks are the planned targets. The Zitarget was reached on the right side, but positioned $6 \mathrm{~mm}$ medial on the left side. Yellow arrow indicates the most distal contact position on the left side. b) Microcirculation (perfusion) as recorded on the left and right sides. c) Total light intensity (TLI) reflecting tissue greyness. The big dip 3-5 mm from the target on the left side shows that the probe passed a darker structure compared to surrounding tissue, this corresponded to the lateral thalamus, and the small dip beyond the target corresponded to the red nucleus (Rn) when comparing with the Schaltenbrandt atlas.

Figure 3. a) Example of very high blood flow values (> 300 a.u.) -6.5 and $-6.0 \mathrm{~mm}$ from the GPitarget on the right side compared to the left side, and b) the corresponding total light intensity (TLI) curves.

Figure 4. Example of raw data of the microcirculation (perfusion in arbitrary units [a.u.], orange) and total backscattered light (TLI, blue) in the vicinity to an STN-target. It is clearly seen that the perfusion changes for each $0.5 \mathrm{~mm}$ step. The calculated mean perfusion (Perf), peak-to-peak (PP) and heart rate (HR) for each recording and each site is marked at the top, and the specific measurement sites as distances to the target at the bottom. A high perfusion spot is seen $+2 \mathrm{~mm}$ beyond the STN target. 
Figure 5. Box plots comparing a) microvascular blood flow (perfusion) and b) total light intensity (TLI) data in the target areas STN $(n=23)$, Vim $(n=12)$ and GPi $(n=9)$. Perfusion data are significantly different while TLI data are not (see Table 1). The boxplots as defined by MATLAB $^{\circledR}$ show Q1 (25\%) to Q3 (75\%) percentiles. The median values are marked with a line in the box for each data set and whiskers are drawn up/down to the closest outliers.

Figure 6. Plots of perfusion (orange) and TLI (blue) recorded at $0.5 \mathrm{~mm}$ resolution the last 10 $\mathrm{mm}$ along a Vim trajectory. A sulci is passed $-50 \mathrm{~mm}$ from the target and at this site TLI is very low at the same time as the blood flow is increasing to a very high level. A TLI peak is seen +0.5 $\mathrm{mm}$ beyond the target, possibly relating to a very thin lamina in the thalamus. At this spot the blood flow is low. Calculated average blood flow (Perf, $\mathrm{m} \pm \mathrm{s}$.d), peak-to peak (P-P), heart rate (HR) and TLI for the two site $(-50 \mathrm{~mm}$ and $+0.5 \mathrm{~mm})$ are presented together with the screen dumps from the recorded signals. 


\section{References}

1. Hemm, S. and K. Wårdell, Stereotactic implantation of deep brain stimulation electrodes: a review of technical systems, methods and emerging tools. Med Biol Eng Comput, 2010. 48(7): p. 611-24.

2. Johnson, M.D., H.H. Lim, T.I. Netoff, A.T. Connolly, N. Johnson, A. Roy, A. Holt, K.O. Lim, J.R. Carey, J.L. Vitek and B. He, Neuromodulation for brain disorders: challenges and opportunities. IEEE Trans Biomed Eng, 2013. 60(3): p. 610-24.

3. Hariz, M., P. Blomstedt and L. Zrinzo, Future of brain stimulation: new targets, new indications, new technology. Mov Disord, 2013. 28(13): p. 1784-92.

4. Zrinzo, L., F. Yoshida, M.I. Hariz, J. Thornton, T. Foltynie, T.A. Yousry and P. Limousin, Clinical safety of brain magnetic resonance imaging with implanted deep brain stimulation hardware: large case series and review of the literature. World Neurosurg, 2011. 76(1-2): p. 164-72; discussion 69-73.

5. Gross, R.E., P. Krack, M.C. Rodriguez-Oroz, A.R. Rezai and A.L. Benabid, Electrophysiological mapping for the implantation of deep brain stimulators for Parkinson's disease and tremor. Mov Disord, 2006. 21(S14): p. S259-S283.

6. Zrinzo, L., T. Foltynie, P. Limousin and M.I. Hariz, Reducing hemorrhagic complications in functional neurosurgery: a large case series and systematic literature review. J Neurosurg, 2012. 116(1): p. 84-94.

7. Zrinzo, L. and M. Hariz, Impedance recording in functional neurosurgery, in Textbook of stereotactic and functional neurosurgery, P.L. Gildenberg, A.M. Lozano, and R. Tasker, Editors. 2008.

8. Antonsson, J., O. Eriksson and K. Wårdell, Radio frequency electrode system for optical lesion size estimation in functional neurosurgery. J Biomed Opt, 2005. 10(3): p. 034020.

9. Wårdell, K., P. Blomstedt, J. Richter, J. Antonsson, O. Eriksson, P. Zsigmond, A.T. Bergenheim and M.I. Hariz, Intracerebral microvascular measurements during deep brain stimulation implantation using laser Doppler perfusion monitoring. Stereotact Funct Neurosurg, 2007. 85(6): p. 279-86.

10. Qian, Z., S. Sunder, G. Yeqing, C. Giller and H. Liu, "Look-ahead distance" of a fiber probe used to assist neurosurgery: Phanton and Monte Carlo study. Optics express, 2003. 11: p. 1844-1855. 
11. Johansson, J.D., I. Fredriksson, K. Wårdell and O. Eriksson, Simulation of reflected light intensity changes during navigation and radio-frequency lesioning in the brain. J Biomed Opt, 2009. 14(4): p. 044040.

12. Johansson, J.D., P. Blomstedt, N. Haj-Hosseini, A.T. Bergenheim, O. Eriksson and K. Wårdell, Combined Diffuse Light Reflectance and Electrical Impedance Measurements as a Navigation Aid in Deep Brain Surgery. Stereotact Funct Neurosurg, 2009. 87(2): p. 105-113.

13. Giller, C.A., H.L. Liu, P. Gurnani, S. Victor, U. Yasdani and D.C. German, Validation of a near-infrared probe for detection of thin intracranial white matter structures. J Neurosurg, 2003. 98: p. 1299-1306.

14. Antonsson, J., O. Eriksson, P. Blomstedt, A.T. Bergenheim, I.H. M, J. Richter, P. Zsigmond and K. Wårdell, Diffuse reflectance spectroscopy measurements for tissue-type discrimination during deep brain stimulation. J Neural Eng, 2008. 5(2): p. 185-90.

15. Giller, C.A., H. Liu, D.C. German, D. Kashyap and R.B. Dewey, A stereotactic near-infrared probe for localization during functional neurosurgical procedures: further experience. J Neurosurg, 2009. 110(2): p. 263-73.

16. Johansson, J.D. and K. Wårdell, Intracerebral quantitative chromophore estimation from reflectance spectra captured during deep brain stimulation implantation. J Biophotonics, 2013. 6(5): p. 435-45.

17. Wårdell, K., P. Zsigmond, J. Richter and S. Hemm, Relationship between laser Doppler signals and anatomy during deep brain stimulation electrode implantation toward the ventral intermediate nucleus and subthalamic nucleus. Neurosurgery, 2013. 72(2 Suppl Operative): p. ons127-40.

18. Nilsson, G.E., T. Tenland and P.A. Öberg, Evaluation of a laser Doppler flowmeter for measurement of tissue blood flow. IEEE Trans Biomed Eng, 1980. 27(10): p. 597-604.

19. Strömberg, T., K. Wårdell, M. Larsson and S. Salerud, Laser Doppler monitoring and imaging techniques, in Biomedical Photonics Handbook, T. Vo-Dinh, Editor. 2014, CRC. p. 435-459.

20. Jacobs, P., Sterrad 100S, Sterilisation system. Johnson \& Johnson, 2006.

21. Terao, T., H. Takahashi, F. Yokochi, M. Taniguchi, R. Okiyama and I. Hamada, Hemorrhagic complication of stereotactic surgery in patients with movement disorders. J Neurosurg, 2003. 98(6): p. 1241-6. 
22. Ben-Haim, S., W.F. Asaad, J.T. Gale and E.N. Eskandar, Risk factors for hemorrhage during microelectrode-guided deep brain stimulation and the introduction of an improved microelectrode design. Neurosurgery, 2009. 64(4): p. 754-62; discussion 762-3.

23. Lemaire, J.J., L. Sakka, L. Ouchchane, F. Caire, J. Gabrillargues and J.M. Bonny, Anatomy of the human thalamus based on spontaneous contrast and microscopic voxels in high-field magnetic resonance imaging. Neurosurgery, 2010. 66(3 Suppl Operative): p. 161-72.

24. Hillman, E.M., Optical brain imaging in vivo: techniques and applications from animal to man. J Biomed Opt, 2007. 12(5): p. 051402.

25. Warden, M.R., J.A. Cardin and K. Deisseroth, Optical neural interfaces. Annu Rev Biomed Eng, 2014. 16: p. 103-29.

26. Wårdell, K., A. Jakobsson and G.E. Nilsson, Laser Doppler perfusion imaging by dynamic light scattering. IEEE Trans Biomed Eng, 1993. 40(4): p. 309-16.

27. Raabe, A., D. Van De Ville, M. Leutenegger, A. Szelenyi, E. Hattingen, R. Gerlach, V. Seifert, C. Hauger, A. Lopez, R. Leitgeb, M. Unser, E.J. Martin-Williams and T. Lasser, Laser Doppler imaging for intraoperative human brain mapping. Neuroimage, 2009. 44(4): p. 1284-9.

28. Dreier, J.P., S. Major, A. Manning, J. Woitzik, C. Drenckhahn, J. Steinbrink, C. Tolias, A.I. Oliveira-Ferreira, M. Fabricius, J.A. Hartings, P. Vajkoczy, M. Lauritzen, U. Dirnagl, G. Bohner and A.J. Strong, Cortical spreading ischaemia is a novel process involved in ischaemic damage in patients with aneurysmal subarachnoid haemorrhage. Brain, 2009. 132(Pt 7): p. 1866-81.

29. Rejmstad, P., G. Åkesson, O. Åneman and K. Wårdell, A laser Doppler system for monitoring cerebral microcirculation: implementation and evaluation during neurosurgery. Med Biol Eng Comput, p 1-9, June 24, 2015. [Epub ahead of print] 
Table 1. $p$-values for comparison of microvascular blood flow (perfusion) and total light intensity (TLI) representing tissue greyness in different regions $(10,5,2.5 \mathrm{~mm}$ and target), and between the three target structures (STN, Vim and GPi)

\begin{tabular}{|l|c|c|c|c|c|c|c|c|}
\hline & \multicolumn{4}{|c|}{ Perfusion } & \multicolumn{5}{c|}{ TLI } \\
\cline { 2 - 9 } & $\mathbf{- 1 0 . 0}$ to $\mathbf{0}$ & $\mathbf{- 5 . 0}$ to 0 & $\mathbf{- 2 . 5}$ to $\mathbf{0}$ & $\mathbf{- 0 . 5}$ to $+\mathbf{0 . 5}$ & $\mathbf{- 1 0 . 0}$ to $\mathbf{0}$ & $\mathbf{- 5 . 0}$ to $\mathbf{0}$ & $\mathbf{- 2 . 5}$ to $\mathbf{0}$ & $\mathbf{- 0 . 5}$ to $+\mathbf{0 . 5}$ \\
\hline Vim-GPi & $<0.001$ & $<0.001$ & $<0.001$ & $<0.005$ & $<0.005$ & 0.108 & $<0.010$ & 0.193 \\
\hline Vim-STN & $<0.001$ & $<0.001$ & $<0.001$ & $<0.050$ & $<0.005$ & $<0.005$ & $<0.050$ & 0.285 \\
\hline STN-GPi & 0.076 & 0.311 & $<0.005$ & $<0.050$ & $<0.001$ & $<0.005$ & $<0.005$ & 0.071 \\
\hline
\end{tabular}




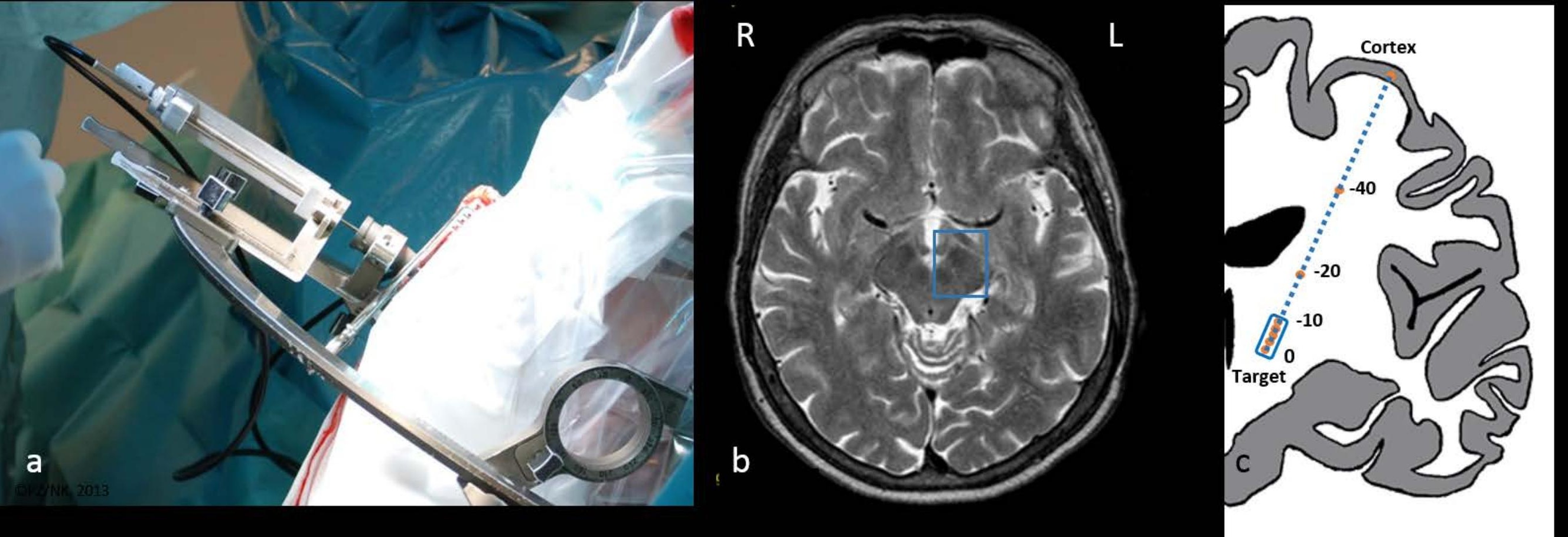




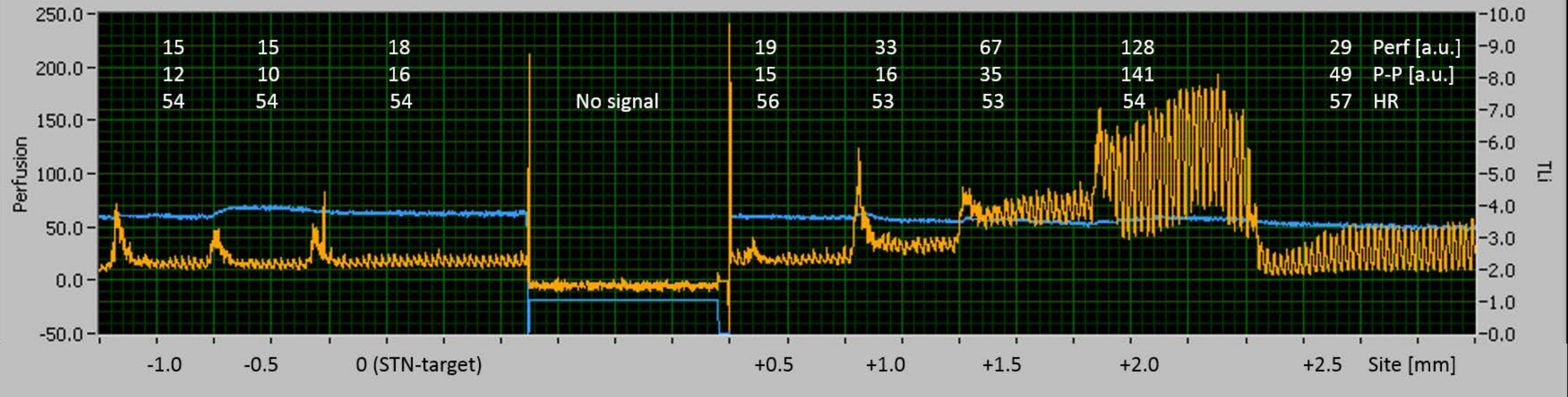



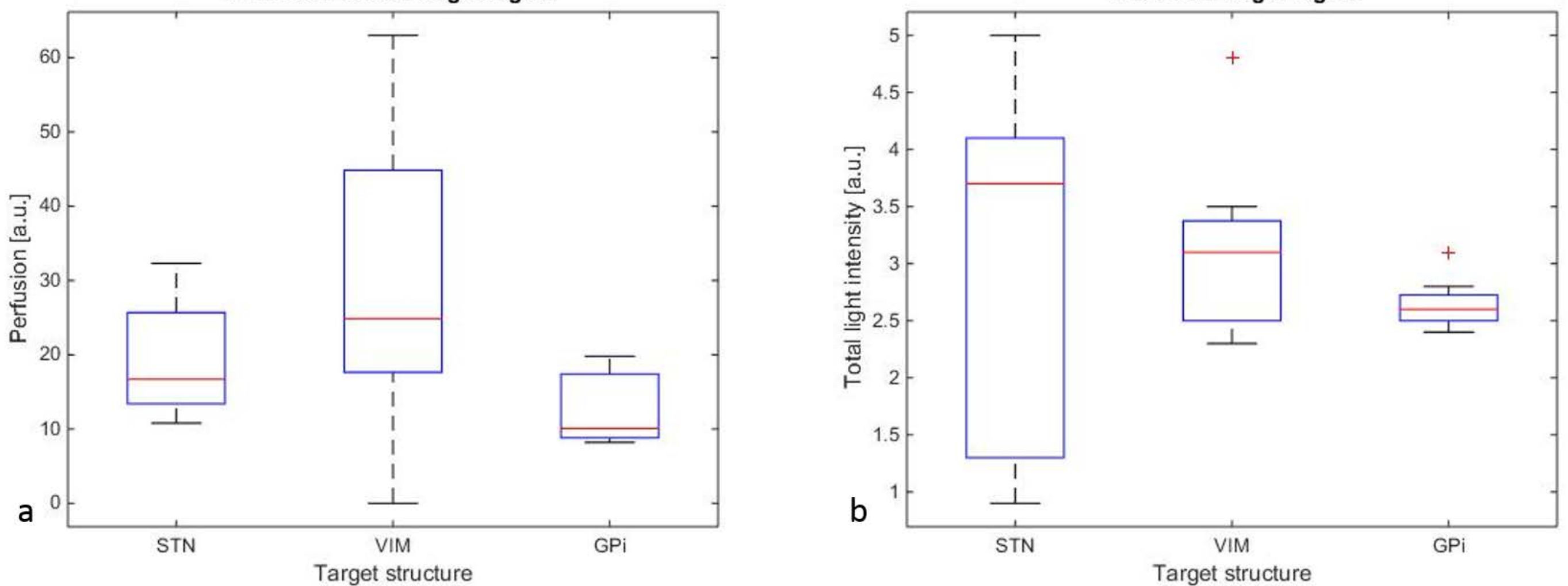


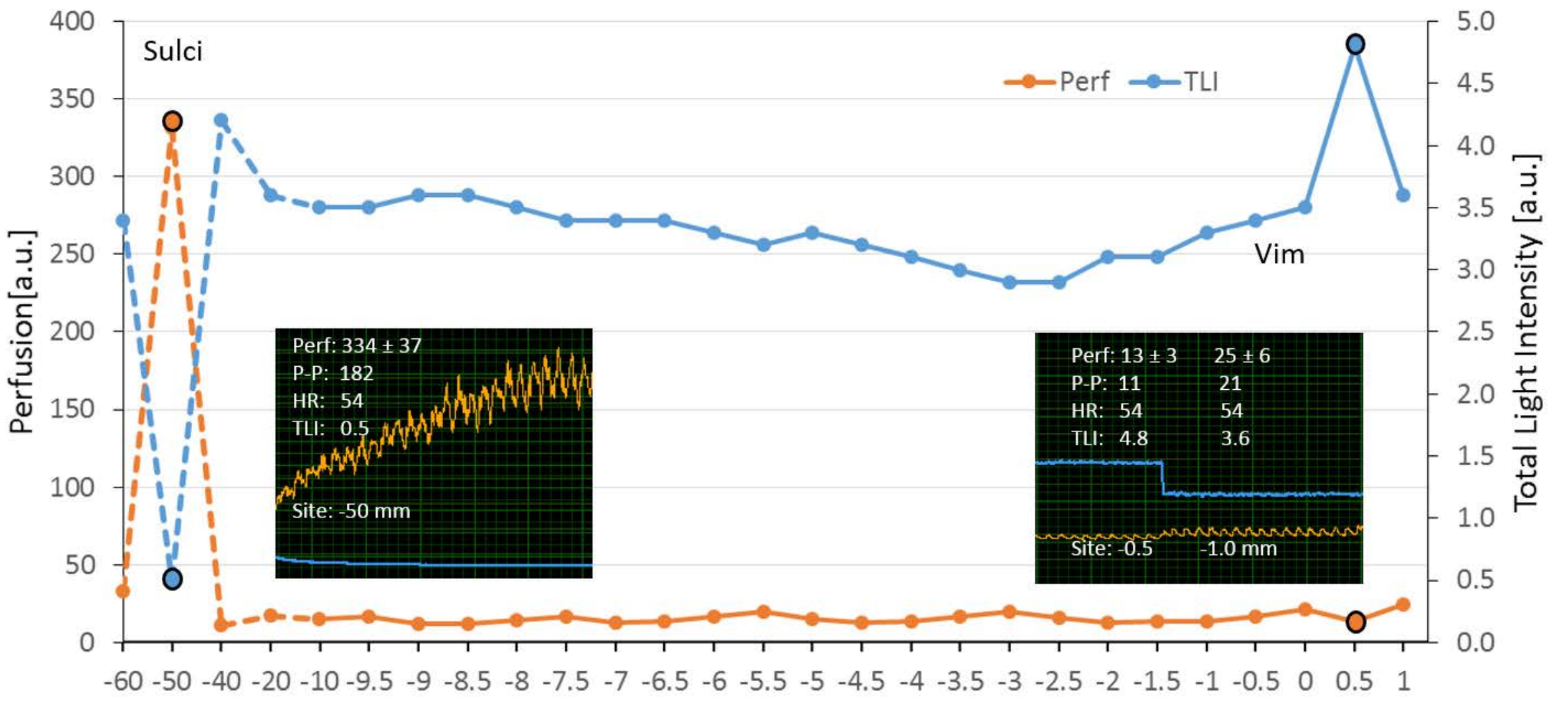

Measurement Site $[\mathrm{mm}]$ 